\title{
Traceable Power Measurement of LTE Signals
}

\author{
Soumya Dash ${ }^{1,2, a}$, Frederic Pythoud ${ }^{1}$, Pascal Leuchtmann ${ }^{2}$ and Juerg Leuthold ${ }^{2}$ \\ ${ }^{1}$ Federal Institute of Metrology METAS, EMC Laboratory, Lindenweg 50, 3003 Bern, Switzerland \\ ${ }^{2}$ Institute of Electromagnetic Fields IEF, ETH Zurich, Gloriastrasse 35, 8092 Zurich, Switzerland
}

\begin{abstract}
Résumé. Cette contribution montre comment effectuer des mesures traçables de l'intensité du rayonnement LTE (Long Term Evolution, ou téléphonie mobile de quatrième génération). Pour pourvoir contrôler que les valeurs limites sont respectées, , il est important de pouvoir mesurer le champ produit par des antennes de téléphonie mobiles. Comme le champ varie suivant le nombre d'utilisateurs connectés, il est important de pouvoir mesurer le "canal de contrôle" dont la puissance est constante. Si pour GSM cela était relativement aisé, pour LTE qui utilise une modulation plus complexe (OFDMA), il faut d'abord démoduler le signal avant de pouvoir le quantifier. Cette contribution présente une méthode qui permet de mesurer de façon traçable les signaux des cellules de référence spécifiques (CSR) d'un signal LTE. Les premières évaluations expérimentales de cette méthode sont également présentées.
\end{abstract}

\section{Introduction and Motivation}

Traceable measurements of the power for mobile communication are important in order to protect the population from excessive levels of non-ionising radiation attributed to a wireless transmission system, like for example mobile base stations. In this paper we propose a method for measuring the maximum power of downlink Long-Term-Evolution (LTE) signals. The proposed method is based on the traceable measurement of the radiated fields produced by the cell-specific reference (CSR) signals.

The current cellular wireless technology rolled out worldwide is the fourth generation of mobile telecommunications technology called LTE. It uses a complex advanced modulation format as Orthogonal Frequency Division Multiple Access (OFDMA) and a multiple antenna technology such as Multiple Input Multiple Output (MIMO), to ensure high data rates, throughput and spectrum efficiencies. A method ensuring the traceability of measurement to the international system (SI) of units is needed for predicting the maximum fields due to LTE signals in indoor and outdoor environments. The principles of these measurements have been proposed in 2012 by METAS [1], without providing the way to achieve full traceability to the SI.

In this contribution we achieve the full traceability of the proposed code selective method. The method is based on scope measurements performed directly at the output of a measuring antenna. The method implements selfdeveloped offline digital signal processing (DSP) demodulation algorithms that include the digital downconversion, timing synchronization, frequency synchronization, phase synchronization and robust LTE cell identification to produce the downlink timefrequency LTE grid. The CSR elements are identified according to the LTE standard, which provides the mapping techniques of these CSR signals to the timefrequency grid depending upon the cell-ID and antenna port.

\section{The Method}

The method is motivated to use the CSR symbols for power measurement of the received signal because the LTE standard [2-4] defines these CSR symbol as the constant reference channel wherein a defined power level is transmitted regardless of weather data traffic is present or weather user device is connected. Hence they are of particular interest in the context of emission measurement of the non-ionizing radiation. Moreover the CSR signals are unique for a given antenna port and in case of multiple antennas (MIMO) configuration the resource element used for transmission of CSR on any of the antenna ports are not be used for any transmission on any other antenna port and they are set to zero.

\subsection{The setup}

As depicted in Figure 1 the system is composed of a local oscillator, a frequency mixer, a low pass filter, a Digital Storage Oscilloscope (DSO) and self-developed software for both the offline Digital Signal Processing (DSP) and power evaluation of the LTE signals.

\footnotetext{
${ }^{\mathrm{a}}$ Corresponding author: soumya.dash@metas.ch
} 


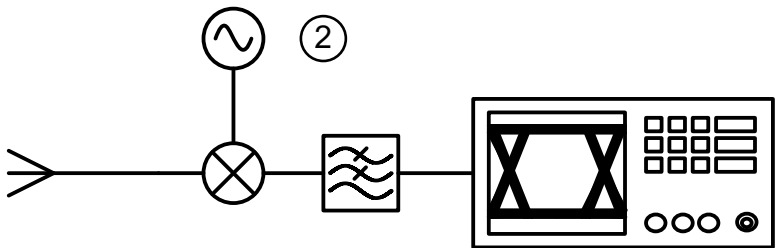

(1) (3) (4) (5)

Figure 1: Schematic representation of the LTE power evaluation hardware module. 1. The input LTE RF signal, 2. Local oscillator, 3. RF mixer, 4. Low pass filter and finally 5.The high speed digital storage oscilloscope

In other words, the input of the measuring antenna ports is first mixed with a stable sine wave in order to produce, after filtering, a signal at the Intermediate Frequency (IF) of typically $50 \mathrm{MHz}$. This IF signal is passed through a low pass filter which eliminates the higher order harmonics and finally the down-converted Radio Frequency (RF) signal is digitized.

Thus, the signal is recorded for a typical duration of $20 \mathrm{~ms}$ at the moment. The duration of the record is only limited by the memory depth of the scope.

Finally, post processing with our own algorithm implemented in MATLAB, provides the power of the CSR signals.

\subsection{The algorithm}

The algorithm is one important part of the traceability of the method. Figure 2 depicts the complex representation of the symbol in a constellation diagram before and after different synchronization and correction steps i.e. Timing synchronization, Frequency correction and Phase correction, implemented in the algorithm. The transparent data processing of the LTE signals guarantees that no disturbing factors, scaling corrections, or any other effects are aliasing the power measurements. Below the algorithm is presented in detail.

\subsubsection{Extraction of In-phase (I) and Quadrature- phase (Q) components}

This first step of the digital algorithm is the extraction of in-phase (I) and in-quadrature (Q) components. The digitized signal represents the LTE signal modulated at an intermediate frequency (IF) of about $50 \mathrm{MHz}$. The I and $\mathrm{Q}$ components are directly obtained using the Digital Down Conversion (DDC) consisting of a multiplication with $\cos \left(2 \pi f_{i f} \cdot t\right)$ and $\sin \left(2 \pi f_{i f} \cdot t\right), \quad f_{i f}$ being the intermediate frequency, and by filtering the result in terms of a root raised cosine filter.

\subsubsection{Resampling of the signal}

The next step in the DSP is resampling of the received signal to the sampling time $T_{s}$, specified in the LTE standards [3] as

$$
T_{s}=\frac{1}{(15000 \times 2048)} \text { seconds }
$$

\subsubsection{Timing synchronization}

According to [5] we implement a correlation receiver for the synchronization of the resampled received signal. The timing synchronization is implemented in the timedomain by using the zero auto-correlation property of the Zadoff Chu sequence [6, 7] present in the Primary Synchronization Signal (PSS) of the LTE signals. The LTE standards define three different Zadoff Chu sequences and only one of the defined sequences is present in the LTE signal of $5 \mathrm{~ms}$. Hence we perform a cross-correlation in between the recorded signal of $r(n)$ and time domain Zadoff Chu sequence $d_{i}(n)$ where $i$ and $n$ denotes the $i^{\text {th }}$ Zadoff Chu sequence and the sample number respectively.

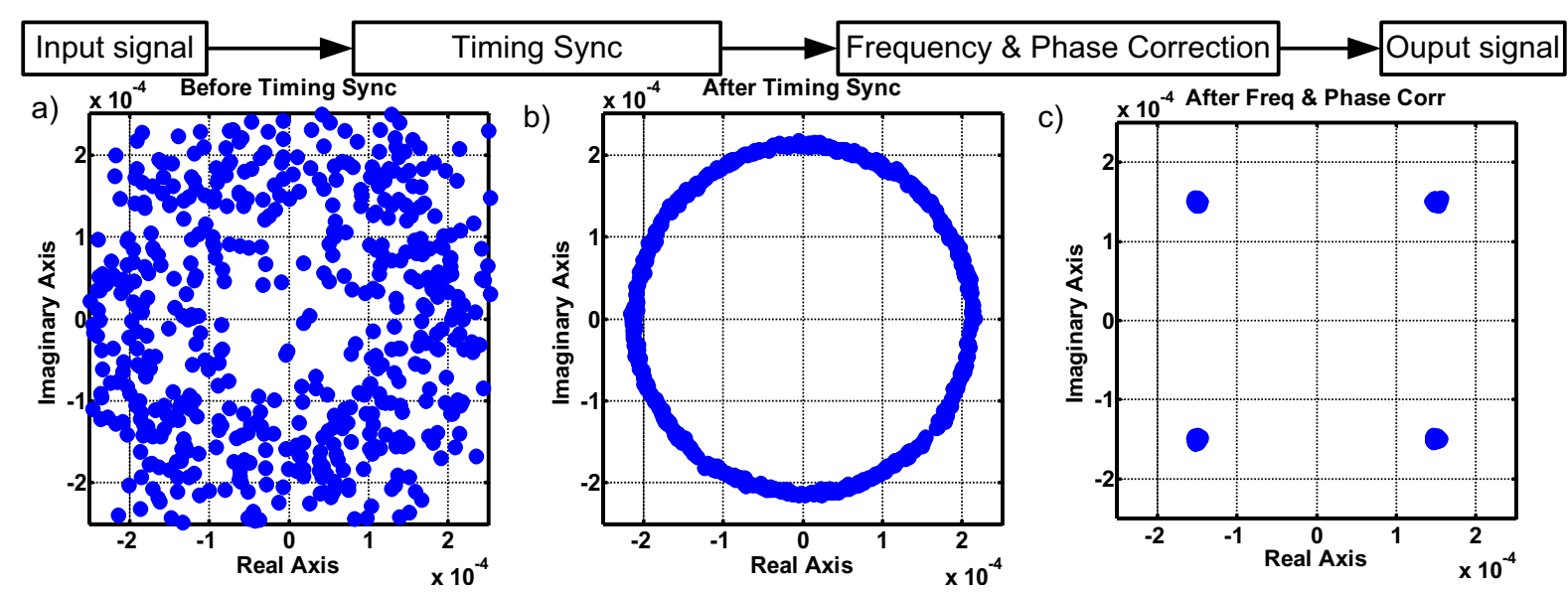

Figure 2 : The constellation diagram of a LTE OFDM symbol with 4-QAM modulation and 1200 active subcarrier. The complex baseband signal is processed with the synchronization steps and the constellation diagram is plotted for (a) before timing synchronization, (b) after timing synchronization and (c) after frequency and phase correction 


$$
c_{i}(n)=\sum_{m=-\frac{L_{P S S}}{2}}^{\frac{L_{P S S}}{2}} d_{i}^{*}(m) r(n+m)
$$

where $L_{P S S}$ denotes the length of the PSS sequence, typically 2048, which corresponds to the size of the FFT for the LTE signals [3]. The cross-correlation is determined for all $n$ values and for all 3 different Zadoff Chu sequences (index $i$ ). The timing $(n)$ and the identification $(i)$ of the correct Zadoff-Chu sequences are clearly identified by the fact that the magnitude of the cross correlation $c_{i}(n)$ is maximum compared to the other correlation terms.

The Figure 2 (a) show the constellation diagram of an OFDM symbol with a 4-QAM modulation and 1200 active subcarriers before timing synchronization and Figure 2 (b) shows the constellation diagram after the correct timing synchronization, where all the points in the constellation lie in a circle and the radius of this circle gives the amplitude of the 4-QAM modulation in the received signal.

\subsubsection{Extraction of symbols in time domain}

Once the timing has been defined precisely, it is quite obvious to extract the symbols in time domain from the time signals. This is performed after removing the cycling prefix of the OFDM modulated signal according to the LTE modulation definition [3].

\subsubsection{Conversion of symbols in the frequency domain}

The symbols in time domain are then converted into the frequency domain in terms of an FFT. We thus get the following resource elements

$$
R E_{l, \text { uncorr }}(k)
$$

representing the uncorrected resource element (real and imaginary value) of the $l^{\text {th }}$ symbol and the $k^{\text {th }}$ subcarrier.

\subsubsection{Frequency correction}

The Zadoff Chu sequence in the frequency domain is considered for this task. The Zadoff Chu sequence is present in the center 62 subcarrier of the PSS signal, center being the DC carrier.

The phase deviation $\gamma$ between the received signal and the reference Zadoff Chu sequence is given as

$$
\begin{aligned}
\gamma_{l_{n}}(k)=\arg \left(R E_{l, \text { uncorr }}^{*}(k) R_{i}(k)\right) \\
-31 \leq k \leq 31, k \neq 0
\end{aligned}
$$

where $n$ is the number of a half-radio-frame and $l_{n}$ being the symbol containing the PSS in the $n^{\text {th }}$ half-radio-frame and $R_{i}(k)$ is the $k^{\text {th }}$ value of the $i^{\text {th }}$ Zadoff-Chu sequence. Here the $i^{\text {th }}$ Zadoff-Chu sequence is determined during the timing synchronization.

The frequency estimate is based on the phase rotation in between the consecutive Zadoff Chu sequence (which is repeated after every $T=5 \mathrm{~ms}$ [3]) is given by

$$
f_{\text {offset }}=\frac{1}{T}\left\{\frac{\Delta \gamma}{2 \pi}\right\}
$$

where

$$
\Delta \gamma=\left\langle\gamma_{l_{n+1}}(k)\right\rangle_{\text {Mean overall } k}-\left\langle\gamma_{l_{n}}(k)\right\rangle_{\text {Mean overall } k}
$$

is the mean difference between the phases of consecutive Zadoff Chu sequences.

The frequency correction is performed by de-rotating the received OFDM , i.e., multiplying it with

$$
e^{-j 2 \pi \cdot f_{\text {offset }} \cdot l /\left(l_{n+1}-l_{n}\right)} ; l_{n} \leq l \leq l_{n+1} .
$$

\subsubsection{Phase correction}

In order to remove resting phase errors in the signals, we perform the following evaluation of the phase deviation $\gamma$. The linear interpolation of the $\gamma_{l_{n}}(k)$ considered as a function of $k$ gives the phase correction for the centre 62 sub-carriers within one symbol. This provides a slope and constant, which are used to extrapolate the phase correction for all the active subcarriers within the same symbol $(k>31$ and $k<-31)$ in the entire bandwidth. The extrapolated phase correction is then applied to all the OFDM symbols in the corresponding half-radio frame of $5 \mathrm{~ms}$.

After having applied both frequency correction and phase correction, we get the following Resource Elements (RE)

$$
R E_{l}(k)
$$

representing the synchronized and corrected resource element (real and imaginary value) of the $l^{\text {th }}$ symbol and the $k^{\text {th }}$ subcarrier. Figure 2 (c) represents the constellation diagram of the frequency and phase corrected OFDM symbol with the 4-QAM modulation.

\subsubsection{Analysis of the Secondary Synchronization Signal}

The LTE signal defines a Secondary Synchronization Signal (SSS) which consists of 168 frequency domain sequences with the length of 62 complex entries [3]. This is present in the OFDM symbol before the symbol with PSS. This sequence is mapped to the 62 sub-carriers located symmetrically around the DC carrier. The sequence is identified by cross-correlation of the 
reference SSS $D_{i}(k)$ where $k$ denotes the element of the $i^{\text {th }}$ SSS sequence.

$$
C_{i}=\sum_{k=-31, k \neq 0}^{31} D_{i}^{*}(k) R E_{l_{n}}(k)
$$

where $R E_{l_{n}}(k)$ is the resource element of a symbol containing the SSS. The magnitude of cross correlator output $C_{i}$ has a large absolute value compared to the other correlation terms which gives the $i^{\text {th }}$ SSS sequence. From the knowledge of the $i$ providing maximum amplitude, we get the $N_{I D}^{(1)}$ representing the physical layer identity in the physical layer cell identity group.

\subsubsection{Finding the Cell Specific Reference signal}

The Cell Specific Reference Signals (CSR) is a sequence defined in the LTE standards [3]. It consists of complex value entries which are mapped on to some of the resource elements of the LTE time-frequency grid depending on the Cell-ID. LTE Downlink (LTE DL) signal defines 504 unique physical layer cell identities $N_{I D}^{(c e l l)}$ which are defined as

$$
N_{I D}^{(c e l l)}=3 \cdot N_{I D}^{(1)}+N_{I D}^{(2)}
$$

where the $N_{I D}^{(1)}$ is computed from the SSS sequence in the range of 0 to 167 and $N_{I D}^{(2)}$ is computed from the PSS sequence with a range of 0 to 2 .

The CSR signals are present on every symbol with index $l=0$ and $l=4$ for one or two antenna port configurations and $l=1$ for higher antenna port configurations. The subcarrier position is given by

$$
k=6 m+\left(v+v_{\text {shift }}\right) \bmod 6
$$

The variables $m, v$ and $v_{\text {shift }}$ are carefully defined within the LTE standard [3]. The refer to the position in the frequency domain for the different cell ID where $m$ corresponds to $m^{\text {th }}$ resource block, the $v$ is given by $p^{\text {th }}$ antenna port and $l^{\text {th }}$ OFDM symbol and the $v_{\text {shift }}$ is given by $N_{I D}^{\text {cell }} \bmod 6$.

\subsubsection{Estimating the power of the CSR}

The power values of the CSR signals are simply obtained by finally estimating the amplitude of the corresponding Resource Elements

$$
\left|R E_{l, c s r}\left(k_{c s r}\right)\right|
$$

Finally the power of the CSR signals extracted from the synchronized LTE time-frequency grid is used for power measurement of the received signals.

\section{Traceability of the algorithm and system}

The absolute calibration of the entire set-up is achieved by characterizing the scope amplitude with an input sine wave of known power. The power of the reference sine wave is obtained in terms of a traceable measurement of its power via a calibrated power meter.

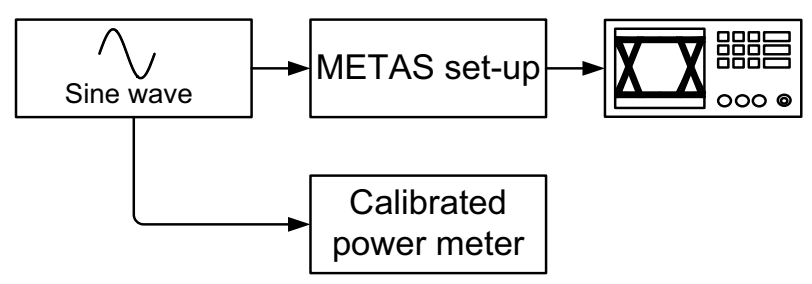

Figure 3 : A sine wave is passed though the set-up developed at METAS for LTE power evaluation and recorded in the DSO as well as measured using a calibrated power meter.

To characterize our set-up we perform a sweep of the sine waves for various frequencies in the neighbourhood of the frequency range of interest then record the waveform in the DSO for $10 \mu \mathrm{sec}$ and measure the power with a calibrated power meter. The measured waveform is evaluated in offline processing to compute the power of the digitized signal.

$$
K(f)=\frac{P_{r e f}(f)}{P_{D S O}(f)}
$$

where $K$ is the power correction factor for the METAS set-up which is given by the ratio the power measured in the power meter $P_{r e f}(f)$ and the power computed using the DSO recording $P_{D S O, f}$ for various frequencies $f \in\left[f_{1}, f_{n}\right]$. This factor can be used as long as the set-up is unchanged to correct the amplitude of the resource elements previously determined.

\section{Experimental Set-up}

To further test the developed algorithm we performed an experiment with Rohde \& Schwarz SMW 200A LTE signal generator. Here the Rohde \& Schwarz SMQ 03B signal generator is used as a local oscillator. The Figure 4 depicts the experimental set-up.wherein the R\&S LTE signal generator outputs RF signals in single channel for SISO configuration and dual channel for the $2 \times 2 \mathrm{MIMO}$ configuration. The dual channel RF output of the signal generator is combined with an Agilent RF power combiner and then mixed with the RF signal from the local oscillator in the mini-circuit RF mixer to produce the IF signal. This IF signal is then passed through a mini-circuit low pass filter to eliminate the higher harmonics and finally measured in the Agilent DSO.

The R\&S LTE signal generator has an inbuilt LTE fading channel emulator which implements different LTE propagation model as defined in the LTE standards 
$[4,8,9]$ for both SISO and MIMO configuration. These different fading models are further used to test the algorithm in different scenarios.

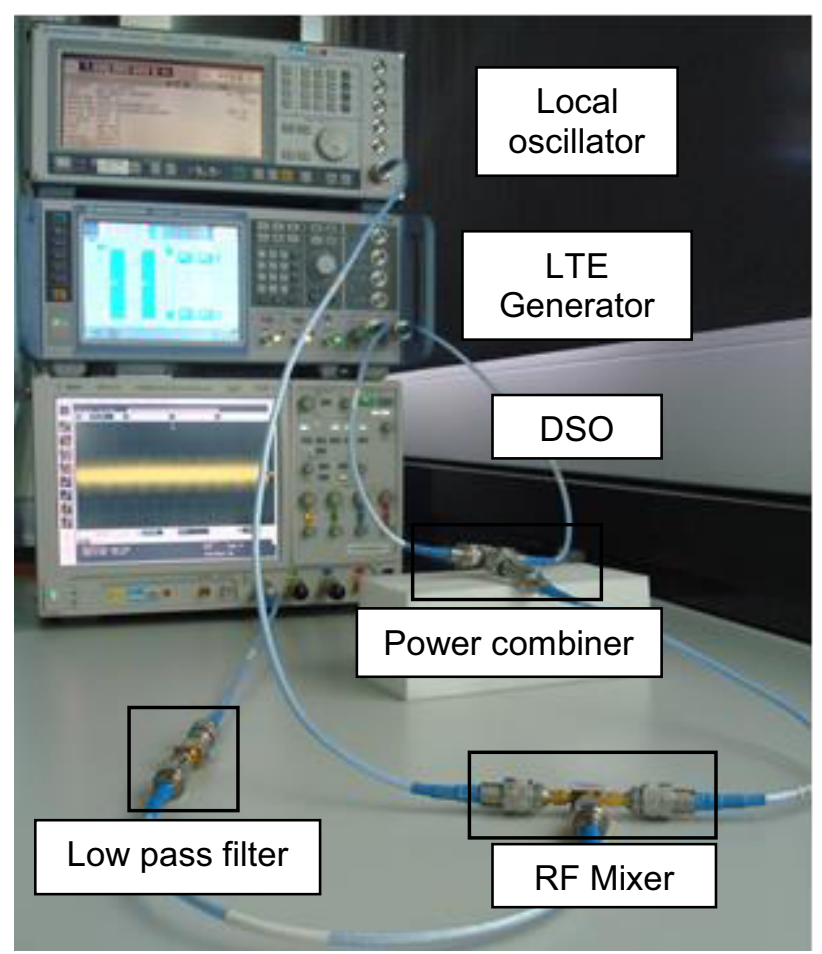

Figure 4 : Experimental set-up for the relative LTE reference channel power measurement of the Rohde \& Schwarz SMW200A using the scope measurement.

The parameters configured in the LTE signal generator and the local oscillator is listed in Table 1. The LTE signal was set to a carrier frequency of $950 \mathrm{MHz}$ and the local oscillator was set to $1 \mathrm{GHz}$ which corresponds to an IF of $50 \mathrm{MHz}$. The reference signal power corresponds to the power level set for the CSR signal and the RS power per RE relative to level display corresponds to the assigned CSR symbol power with respect to the set reference signal power. The local oscillator was set at $10 \mathrm{dBm}$ output power to meet the required power level for the RF mixer. Measurements were done for both conducted SISO and conducted MIMO configuration.

Table 1 : Experimental set-up parameters for the LTE power evaluation of the R\&S SMW 200A

\begin{tabular}{|c|c|}
\hline Experimental set-up parameters & values \\
\hline LTE transmission mode & $\begin{array}{c}\text { FDD mode (Type 1) } \\
\text { 2 Antenna MIMO }\end{array}$ \\
\hline Carrier frequency (A \& B) & $950 \mathrm{MHz}$ \\
\hline RF power level (A \& B) & $0 \mathrm{dBm}$ (A \& B). \\
\hline Channel bandwidth & $20 \mathrm{MHz}$ \\
\hline Sampling frequency & $30.72 \mathrm{MHz}$ \\
\hline
\end{tabular}

\begin{tabular}{|c|c|}
\hline FFT Size & 2048 \\
\hline Cell ID & 1 \\
\hline $\begin{array}{c}\text { Reference signal power } \\
\text { RS power per RE relative to level } \\
\text { display }\end{array}$ & $0 \mathrm{~dB}$ \\
\hline $\begin{array}{c}\text { Local oscillator frequency } \\
\text { Local oscillator power level }\end{array}$ & $10.79 \mathrm{~dB}$ \\
\hline Scope capture time & $20 \mathrm{GH}$ \\
\hline
\end{tabular}

\section{Preliminary results}

The scope measurements were processed with the selfdeveloped algorithm where we were successfully able to synchronize the received signal as explained in the section 2.1 using the Zadoff Chu sequence and extract $N_{I D}^{(2)}$ and $N_{I D}^{(1)}$ information and then compute the Cell ID $N_{I D}^{(c e l l)}$. This cell ID provides us the access into the complex time-frequency grid and the CSR symbol. The power of the CSR symbols was used to compute the signal power. The preliminary results with the MIMO and SISO configuration with channel impairment as defined in $[8,9]$ are depicted in the Table 2. For the SISO configuration we used the Channel Quality Indication (CQI) propagation model and the Extended Pedestrian Model A (EPA) whereas for the MIMO configuration we used the EPA model with both low delay spread (EPA Low) and high delay spread (EPA High). The deviation columns reports the deviation between the CSR LTE powers as measured by our system and the CSR as entered into the previously calibrated signal generator. This deviation is of course not an absolute comparison, but a relative one demonstrating the accuracy of the signal generator.

Table 2 : Different measurement scenarios for the LTE power evaluation

\begin{tabular}{|l|c|c|c|}
\hline & $\begin{array}{c}\text { MIMO } \\
\text { configuration }\end{array}$ & $\begin{array}{c}\text { Fading type } \\
{[8,9]}\end{array}$ & $\begin{array}{c}\text { Deviation } \\
{[\mathrm{dB}]}\end{array}$ \\
\hline Scenario 1 & SISO & No Fading & 0.01 \\
\hline Scenario 2 & SISO & CQI 5Hz & 0.04 \\
\hline Scenario 3 & SISO & EPA 5Hz & 0.08 \\
\hline Scenario 4 & MIMO (2×2) & No Fading & 0.02 \\
\hline Scenario 5 & MIMO $(2 \times 2)$ & $\begin{array}{c}\text { EPA 5Hz } \\
(\text { Low })\end{array}$ & 0.2 \\
\hline
\end{tabular}




\begin{tabular}{|c|c|c|c|}
\hline Scenario 6 & MIMO $(2 \times 2)$ & $\begin{array}{c}\text { EPA 5Hz } \\
(\text { High })\end{array}$ & 0.3 \\
\hline
\end{tabular}

Figure 5 depicts the LTE grid after the synchronization from port 0 of a $2 \times 2$ MIMO configuration for 72 OFDM symbols and center 72 active sub-carriers excluding the center DC carrier. Each cell in the grid represents a resource element. The cell in red is an occupied resource element and the ones in blue are empty resource elements. The blue colored cell spread over the entire grid corresponds to the empty $\mathrm{RS}_{1}$ which are CSR symbols reserved for transmission from antenna port 1 .

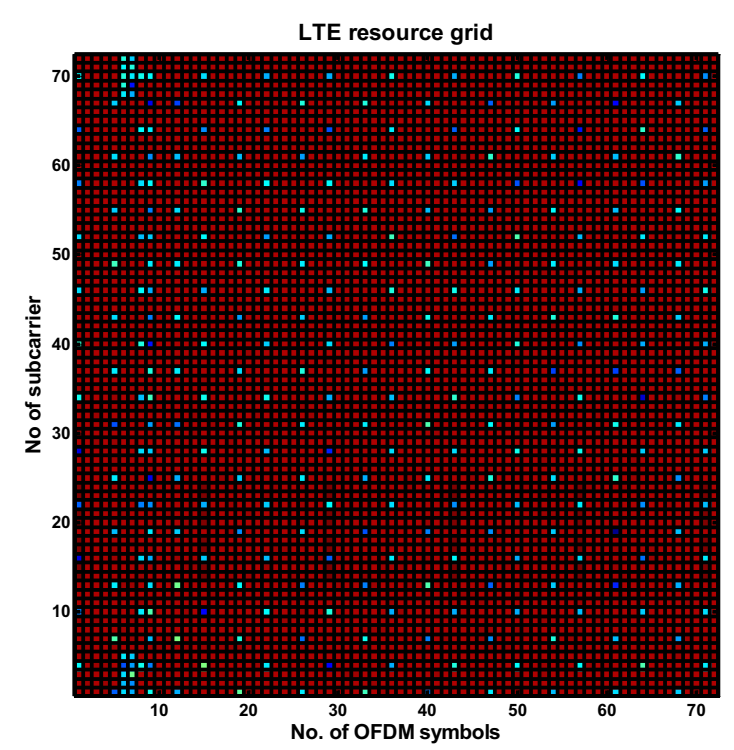

Figure 5: A sample representation of the completely filled LTE time-frequency grid generated from the LTE signal from the port 0 of a $2 \times 2$ MIMO configuration for 72 OFDM symbols and center 72 subcarriers after the offline DSP, including the time, frequency and phase synchronization as described in the section 2.

From Table 2 we can conclude that for no fading scenario the algorithm performs extremely well for SISO and MIMO configuration with deviations of $0.01 \mathrm{~dB}$ and $0.02 \mathrm{~dB}$ respectively. The algorithm is more resilient to the fading scenarios in SISO configuration compared to MIMO configuration. We recorded deviations of $0.04 \mathrm{~dB}$ and $0.08 \mathrm{~dB}$ for CQI $5 \mathrm{~Hz}$ and EPA $5 \mathrm{~Hz}$ fading scenarios respectively in SISO configuration. Similarly we recorded uncertainties of $0.2 \mathrm{~dB}$ and $0.3 \mathrm{~dB}$ for the EPA $5 \mathrm{~Hz}$ slow fading and EPA $5 \mathrm{~Hz}$ fast fading respectively in MIMO configuration.

\section{Discussion and conclusion}

Theses preliminary results show a good agreement between the power "measured" by our system with the power "setup" into the signal generator. The SISO scenarios have maximum deviations of $0.08 \mathrm{~dB}$, as the MIMO scenario show typical deviations of up to $0.3 \mathrm{~dB}$ in case of fading. In case of fading (simulated by the signal generator), it is clear that even the CSR signals are not very stable and that the statistical fluctuations of the signal provide additional uncertainty to the measurements. The work will be continued to better understand these deviations and to provide the most accurate estimate of the power of LTE signals even in complex situations with fading and MIMO.

\section{Acknowledgements}

The research leading to the results described in this paper is part of the European Metrology Research Program EMRP IND 51 "MORSE" (Metrology for optical and RF communication systems). The EMRP is jointly funded by the EMRP participating countries within EURAMET and the European Union.

\section{References}

1. Pythoud, F. and B. Mühlemann, Measurement Method for LTE Base Stations. 2012, Federal Office of Metrology METAS: Bern, Switzerland. p. 16.

2. ETSI, LTE; Evolved Universal Terrestrial Radio Access (E-UTRA); Physical layer procedures (3GPP TS 36.213 version 9.2.0 Release 9), in 3GPP TS 136.213. 2010: Sophia Antipolis Cedex - FRANCE. p. 82-82.

3. ETSI, LTE; Evolved Universal Terrestrial Radio Access (E-UTRA); Physical channels and modulation (3GPP TS 36.211 version 11.5.0 Release 11), in 3GPP TS 136.211. 2014: Sophia Antipolis Cedex FRANCE. p. 122-122.

4. ETSI, LTE; Evolved Universal Terrestrial Radio Access (E-UTRA); Base Station (BS) radio transmission and reception

(Release 12), in 3GPP TS 136.104. 2014: Sophia Antipolis Cedex - FRANCE. p. 152.

5. Yingming, T., et al., Cell search in 3GPP long term evolution systems. Vehicular Technology Magazine, IEEE, 2007. 2(2): p. 23-29.

6. Beyme, S. and C. Leung, Efficient computation of DFT of Zadoff-Chu sequences. Electronics Letters, 2009. 45(9): p. 461-463.

7. Wiki, Zadoff-Chu sequence.

8. ETSI, 3rd Generation partnership Project; Technical Specification Group Radio Access netowrk; Spatial Channel Model for Multiple Input and Multiple Output (MIMO) simulations (Release 12), in 3GPP TR 25.996. 2014: Sophia Antipolis Cedex - FRANCE.

9. ETSI, 3rd Generation partnership Project; Technical Specification Group Radio Access netowrk; Universal Terrestrial Radio Access (UTRA) and Evolved Universal Terrestrial Radio Access (E-UTRA); Verification of radiated multi-antenna reception performance of user equipment (UE) (Release 12), in 3GPP TR 37.977. 2014: Sophia Antipolis Cedex FRANCE. 